\title{
Prevalencia de Anomalías de Pie en Niños de Enseñanza Básica de Entre 6 a 12 Años, de Colegios de la Ciudad de Arica-Chile
}

\author{
Prevalence of Foot Anomalies in Schoolchildren between \\ 6 and 12 Years Old of Elementary Education from Arica-Chile
}

Omar Espinoza-Navarro; María Olivares Urquieta; Paulina Palacios Navarrete \& Noelia Robles Flores

ESPINOZA-NAVARRO, O.; OLIVARES, U. M.; PALACIOS, N. P. \& ROBLES, F. N. Prevalencia de anomalías de pie en niños de enseñanza básica de entre 6 a 12 años, de colegios de la ciudad de Arica-Chile. Int. J. Morphol., 31(1):162-168, 2013.

RESUMEN: Los problemas ortopédicos del pie en el niño son los más frecuentes en esta época de la vida. Por esto, es importante hacer la diferenciación entre un pie normal y un pie alterado. El objetivo fue determinar la prevalencia de pie plano y pie cavo en alumnos de enseñanza básica de Arica. 420 alumnos (210 niñas y 210 niños) de 3 diferentes tipos de colegios de enseñanza básica de la ciudad de Arica, en edades de entre 6 a 12 años, aparentemente sanos, fueron medidos. A todos los sujetos en estudio se les realizó una evaluación podoscópica de la huella plantar desde el plano anteroposterior. A los padres, apoderados y profesores se les aplicó una encuesta sobre el grado de conocimiento y las implicancias de estas anomalías. Los resultados muestran en los niños varones una prevalencia de un $31,6 \%$ para pie plano y un $11,6 \%$ para pie cavo, en relación a un $56,8 \%$ de niños con pie normal. En las niñas los valores indican un 24,3\% para pie plano y un $14,4 \%$ para pie cavo. El peso y el índice de masa corporal presentan una correlación positiva en la expresión de anomalías de pie en la población estudiantil de Arica-Chile. El Colegio "Regimiento Rancagua", de tipo municipal, gratuito, presenta los más altos índices de anomalías de pie (45\%), Colegio particular (43\%) y el Colegio particular subvencionado, los menores índices con un valor de 35\%. En el análisis de las encuestas se observa que padres a apoderados tienen pocos conocimientos sobre estas alteraciones y sus consecuencias en la postura. Pie plano es la anomalía de mayor conocimiento por padres y los profesores. Se concluye que pie plano presenta mayor prevalencia en la población estudiantil de Arica, con un $28 \%$ y pie cavo una prevalencia total de un $13 \%$.

PALABRAS CLAVE: Alteraciones posturales; Educación básica; Niños; Arica-Chile.

\section{INTRODUCCIÓN}

A nivel general podemos definir el pie como una compleja estructura de huesos, músculos y tejidos conectivos que corresponde a la parte de la extremidad inferior que comienza a partir de los tobillos hacia abajo, esencial para la posición bípeda humana y la locomoción (Zurita \& Cabe1lo, 2002; Didia, 2011).

El control de la postura o equilibrio corporal significa mantener el centro de gravedad con una fuerte base de sustentación y la integración de varios sistemas, bajo el control del sistema nervioso, incorporando los órganos vestibulares, cerebelo, vías visuales, propiocepción y sensibilidad superficial y profunda. La expresión de la marcha es por definición la pérdida y recuperación alterna del equilibrio, por esencia es una de las actividades más importantes de la vida diaria (Ibañez et al., 2008; Espinoza-Navarro, et al., 2009).

Los pediatras suelen encontrar una gran variedad de alteraciones en la postura de las piernas y de los pies, desde el período de nacimiento hasta la adolescencia. Los problemas ortopédicos del pie en el niño son los más frecuentes en esta época de la vida. Por este motivo es importante hacer la diferenciación entre un pie normal y un pie alterado que necesita tratamiento del especialista en forma precoz (El et al., 2006; Vasconcellos et al., 2011). 
Actualmente, las anomalías de pie plano y pie cavo son bastantes comunes. Si estas anomalías no son detectadas a tiempo puede traer consecuencias a nivel de pie, tobillo, rodilla, cadera, columna vertebral y hombro.

El pie plano corresponde a la disminución o desaparición de la bóveda plantar (Viladot \& Rochera, 2009), configurada por la base del primer y el quinto metatarsiano y el apoyo del calcáneo. Esta anomalía puede ser de origen congénito o adquirido. Los niños pequeños de 4 a 5 años presentan una imagen de pie aplanado, debido a una bolsa adiposa (grasa), que protege las estructuras cartilaginosas de lo que será el pie en un futuro cuando este termine su desarrollo.

En los adolescentes causa cansancio y esguinces repetidos en los tobillos (articulación crurotalar). Lesiones que en general son toleradas, ya que se niegan a realizar un tratamiento con plantillas u otros, por temor a ser ridiculizados por sus pares. Siendo esta la causa mayoritaria que no exista una preocupación por esta malformación en los pies (El et al.).

En los adultos el pie plano conlleva a molestias mayores de origen articular, y con el transcurso del tiempo hace que dichas estructuras óseas, que no trabajan en posición y alineamiento adecuado, produzcan artrosis a nivel articular de pie y tobillo, presentando dolores y molestias primero ocasionales y posteriormente permanente (Larrosa Padró \& Mas Moliné, 2003).

El pie cavo es aquella deformidad del pie que presenta una acentuación excesiva de la bóveda plantar, y una desviación del retropié en varo o en valgo, puede ser de origen neurológico, congénito o por enfermedades osteoarticulares, si no es corregida tempranamente en las primeras etapas de la niñez, puede ser irreversible y puede causar dolores muy intensos a nivel de los pies, tobillos, rodillas, cadera, columna vertebral y hombro (Ledoux et al., 2006).

Esta patología se asocia a una desviación en varo del calcáneo y retracción de los dedos (dedos en garra). Es una patología rara en los niños, y la sintomatología se hace presente en la adolescencia y en el adulto. El dolor es debido a que el peso del cuerpo es soportado por la cabeza de los metatarsianos y por el talón. Alrededor del $80 \%$ de los pie cavo en el niño se deben a alteraciones neuromusculares, que en algunos casos son de índole familiar (Nikolaidou \& Boudolos, 2006; Onodera et al., 2008).

El objetivo fue determinar la prevalencia de pie plano y pie cavo en alumnos de enseñanza básica de Arica en mujeres y hombres de entre 6 a 12 años correspondiente a cursos desde $1 .^{\circ}$ a $8 .^{\circ}$ año básico, de tres diferentes tipos de establecimientos educacionales de la ciudad de Arica.

\section{MATERIAL Y MÉTODO}

La presente investigación es de tipo descriptiva, con un estudio exploratorio comparativo. La población corresponde a mujeres y hombres de $1 .^{\circ}$ a $8 .^{\circ}$ año básico, en edades de entre 6 a 12 años, de tres tipos diferentes de colegios: Escuela "Regimiento Rancagua" (municipal, público y gratuito), Colegio Abraham Lincoln School (particular subvencionado) y Colegio Junior College (particular no subvencionado). De cada colegio fueron seleccionados 140 alumnos. La muestra en estudio estuvo constituida por un total de 420 alumnos, 210 niñas y 210 niños.

Los alumnos fueron pesados y medidos para realizar el cálculo del índice de masa corporal (IMC), según los protocolos indicados por la OMS, aplicando la fórmula $\mathrm{P} / \mathrm{T}^{2}$, clasificándolos en niños de bajo peso, normal, sobrepeso y obesos. Los valores obtenidos se analizaron por las "Normas de evaluación nutricional en niños chilenos de entre 06 a 18 años (Ministerio de Salud de Chile, 2004). Posteriormente se les realizó la evaluación podoscópica, para identificación de malformación del pie (pie plano y pie cavo), mediante la observación fotográfica de la huella plantar desde el plano anteroposterior.

Para la recolección de los datos se utilizaron balanza médica, para determinar el peso de la masa corporal en kg (Tanita, Modelo 2001), cinta métrica, para evaluar la altura del vértex en $\mathrm{cm}$. Las imágenes plantares se realizaron en podoscopio de cristal sólido, con espejo oblicuo y de haz de luz, ocupando la posición bipodálica (Comín et al., 1999).

A padres y apoderados se les aplicó una "Encuesta individual", para determinar el grado de conocimiento del tema en estudio. Los protocolos ocupados de Consentimiento Informado y Asentimiento de los alumnos, fueron aprobados por el Comité de Ética y Bioética de la Universidad de Tarapacá.

Los resultados obtenidos fueron sometidos a un tratamiento estadístico con estadígrafos de tendencia central y test de Student con un grado de significancia de $\mathrm{p} \leq$ 0,05 . 


\section{RESULTADOS}

En la Tabla I, se muestran los valores de talla, peso e IMC, de la población en estudio. Se observa que la talla tanto en niñas como en niños se encuentra dentro de los percentiles de normalidad. Los hombres presentan mayor peso respecto a las mujeres (promedio de $53,2 \mathrm{~kg} \mathrm{y} 50,7 \mathrm{~kg}$, respectivamente). El índice de masa corporal (IMC), en las niñas de 6 a 10 años indica sobrepeso y en las niñas de 11 a 12 años, niveles de normalidad. Los hombres de 6 años presentan un IMC normal. Desde los 7 años a los 12 años los valores indican sobrepeso.

En la Tabla II, se presentan los porcentajes de anomalías de pie, según el sexo y la edad. Los hombres presentan mayores índices de anormalidad para pie plano $(31,6 \%)$ respecto de las mujeres $(24,3 \%)$. Las mujeres presentan mayores índices de anormalidad en pie cavo, con un 14,4\%, respecto a los hombres con un $11,6 \%$. A partir de los 10 años las anomalías de pie, tienden a bajar significativamente en los hombres, mientras que en las mujeres, se muestra una tendencia a mantener los niveles.
La Tabla III, muestra que en la población general las anomalías de pie más prevalente es pie plano, con un $28 \%$ y pie cavo con un $13 \%$. Los más altos índices de anormalidades se presentan desde los 6 a los 8 años con valores sobre el 52\%. Los niños de 7 años presentan valores de $55 \%$. A partir de los 9 años los índices bajan significativamente, presentando valores desde un $38 \%$ a un $28 \%$ a los 12 años.

En la Tabla IV, se muestran los porcentajes de anomalías según el tipo de establecimiento educacional, donde se observa que el colegio municipal "Regimiento Rancagua”, presenta los mayores índices de anormalidad de pie, con un $45 \%$. El Colegio Abraham Lincoln, presenta los menores índices con un $35 \%$ de anormalidad en pie.

La Tabla V registra el conocimiento de los padres, apoderados y profesores de curso sobre las anomalías de pie plano y pie cavo. Se observa que un $53 \%$ de los padres y apoderados ha realizado algún tipo de examen de pie a

Tabla I. Valores de Talla $(\mathrm{cm})$, peso $(\mathrm{kg})$ y cálculo de índice de masa corporal (IMC), mujeres $(\mathrm{n}=210)$ y hombres $(n=210)$ de entre 6 años a 12 años de establecimientos educacionales de Arica-Chile.

\begin{tabular}{lcccccccc}
\hline & \multicolumn{4}{c}{ Hombres } & \multicolumn{3}{c}{ Mujeres } \\
\cline { 2 - 8 } Edad & $\mathbf{n}$ & $\begin{array}{c}\text { Talla }(\mathbf{c m}) \\
\mathbf{\pm D E}\end{array}$ & $\begin{array}{c}\text { Peso }(\mathbf{k g}) \\
\mathbf{D D E}\end{array}$ & $\mathbf{I M C}$ & $\mathbf{n}$ & $\begin{array}{c}\text { Talla }(\mathbf{c m}) \\
\mathbf{\pm D E}\end{array}$ & $\begin{array}{c}\text { Peso (kg) } \\
\pm \mathbf{D E}\end{array}$ & IMC \\
\hline 6 años & 30 & $125( \pm 5,9)$ & $26,2( \pm 4,6)$ & 16,8 & 30 & $123( \pm 5,9)$ & $26,4( \pm 3,5)$ & 17,4 \\
7 años & 30 & $129( \pm 4,9)$ & $31,8( \pm 3,37)$ & 19,1 & 30 & $132( \pm 5,6)$ & $32,5( \pm 7,4)$ & 18,7 \\
8 años & 30 & $129( \pm 8,19)$ & $32,1( \pm 5,72)$ & 19,3 & 30 & $134( \pm 6,6)$ & $33,8( \pm 7,98)$ & 18,8 \\
9 años & 30 & $139( \pm 5,68)$ & $42,2( \pm 9,12)$ & 21,8 & 30 & $138( \pm 7,6)$ & $37,5( \pm 8,39)$ & 19,7 \\
10 años & 30 & $145( \pm 8,85)$ & $43,1( \pm 10,4)$ & 20,5 & 30 & $146( \pm 5,4)$ & $41,1( \pm 9,46)$ & 20,2 \\
11 años & 30 & $147( \pm 6,16)$ & $45,9( \pm 9,06)$ & 21,2 & 30 & $148( \pm 5,7)$ & $45,5( \pm 10,04)$ & 20,8 \\
12 años & 30 & $154( \pm 7,68)$ & $53,2( \pm 8,09)$ & 22,4 & 30 & $153( \pm 7,6$ & $50,7( \pm 8,54)$ & 21,7 \\
Promedio & & & & 20,2 & & & & 19,6 \\
\hline
\end{tabular}

Tabla II. Porcentajes de anomalías de pie (\%), según sexo y edad de población estudiantil (n=420) de Arica-Chile.

\begin{tabular}{lcccccccc}
\hline & \multicolumn{3}{c}{ Hombres } & \multicolumn{4}{c}{ Mujeres } \\
\cline { 2 - 9 } Edad & $\mathbf{n}$ & $\begin{array}{c}\text { Pie normal } \\
(\boldsymbol{\%})\end{array}$ & $\begin{array}{c}\text { Pie plano } \\
(\boldsymbol{\%})\end{array}$ & $\begin{array}{c}\text { Pie cavo } \\
(\boldsymbol{\%})\end{array}$ & $\mathbf{n}$ & $\begin{array}{c}\text { Pie normal } \\
(\boldsymbol{\%})\end{array}$ & $\begin{array}{c}\text { Pie plano } \\
(\boldsymbol{\%})\end{array}$ & $\begin{array}{c}\text { Pie cavo } \\
(\boldsymbol{\%})\end{array}$ \\
\hline 6 años & 30 & 33 & 67 & 0 & 30 & 63 & 26 & 11 \\
7 años & 30 & 37 & 44 & 19 & 30 & 52 & 21 & 28 \\
8 años & 30 & 58 & 30 & 11 & 30 & 38 & 41 & 21 \\
9 años & 30 & 39 & 43 & 17 & 30 & 84 & 8 & 18 \\
10 años & 30 & 81 & 8 & 12 & 30 & 64 & 18 & 18 \\
11 años & 30 & 68 & 14 & 18 & 30 & 66 & 28 & 7 \\
12 años & 30 & 81 & 15 & 4 & 30 & 62 & 28 & 10 \\
Promedio & & 56,8 & 31,6 & 11,6 & & 61,3 & 24,3 & 14,4 \\
\hline
\end{tabular}


sus hijos. Un $78 \%$ de ellos muestra preocupación por el uso de un buen calzado. Los mayores conocimientos se refieren a pie plano con un $65 \%$ y pie cavo con un $46 \%$. Solo un $26 \%$, conoce sobre las políticas de salud respecto a anomalías de pie. Un 55\% dice conocer sobre las consecuencias de no detectar a tiempo anomalías de pie. Respecto a los profesores, el $81 \%$ de ellos a realizado algún tipo de examen de pie a sus alumnos, igualmente conocen más de pie plano que de pie cavo $(63 \%$ y $37 \%$ respectivamente). Solo un $22 \%$ conoce sobre el uso de plantillas correctivas. Todos los profesores encuestados dicen aceptar la ayuda de un especialista en sus clases de educación física.
Tabla III. Porcentajes totales de anomalías de pie (\%), según la edad y el sexo en niños y niñas escolares $(n=420)$ de Arica-Chile.

\begin{tabular}{lcccc}
\hline Edad & n & $\begin{array}{c}\text { Pie normal } \\
(\boldsymbol{\%})\end{array}$ & $\begin{array}{c}\text { Pie plano } \\
(\boldsymbol{\%})\end{array}$ & $\begin{array}{c}\text { Pie cavo } \\
(\boldsymbol{\%})\end{array}$ \\
\hline 6 años & 60 & 33 & 46 & 6 \\
7 años & 60 & 48 & 32 & 23 \\
8 años & 60 & 45 & 36 & 16 \\
9 años & 60 & 62 & 26 & 12 \\
10 años & 60 & 71 & 14 & 15 \\
11 años & 60 & 67 & 21 & 12 \\
12 años & 60 & 72 & 21 & 7 \\
Promedio & & $59(\mathrm{n}=248)$ & $28(\mathrm{n}=113)$ & $13(\mathrm{n}=59)$ \\
\hline
\end{tabular}

Tabla IV. Porcentajes (\%) de anomalías en pie según los diferentes establecimientos educacionales de Arica-Chile.

\begin{tabular}{|c|c|c|c|c|c|c|c|c|c|}
\hline \multirow[b]{2}{*}{ Colegio } & \multicolumn{2}{|c|}{ Pie normal (\%) } & \multicolumn{2}{|c|}{ Pie plano $(\%)$} & \multicolumn{2}{|c|}{ Pie cavo $(\%)$} & \multicolumn{2}{|c|}{ Total $(\%)$} & \multirow[b]{2}{*}{$\mathbf{n}$} \\
\hline & Hombres & Mujeres & Hombres & Mujeres & Hombres & Mujeres & Normal & Anomalías & \\
\hline Junior College & 52,8 & 61 & 36,1 & 22,2 & 11,1 & 16,8 & 57 & 43 & 140 \\
\hline Abraham Lincoln & 56,9 & 72,2 & 37,5 & 25 & 5,6 & 2,8 & 65 & 35 & 140 \\
\hline Regimiento Rancagua & 61,1 & 48,6 & 23,6 & 18 & 15,3 & 33,4 & 55 & 45 & 140 \\
\hline $\mathbf{n}$ & 120 & 128 & 68 & 45 & 29 & 30 & & & 420 \\
\hline
\end{tabular}

Tabla V. Resultados de las encuestas sobre conocimientos de anomalías de pie, aplicadas a los padres y apoderados y profesores de los alumnos en estudio en Arica-Chile.

\begin{tabular}{|c|c|c|c|c|}
\hline \multirow{2}{*}{ Pregunta } & \multicolumn{2}{|c|}{ Padres } & \multicolumn{2}{|c|}{ Profesores } \\
\hline & $\mathbf{S i}$ & No & $\mathbf{S i}$ & No \\
\hline 1.- ¿Ha realizado al gún examen de pie a su hijo-alumno? & $53 \%$ & $47 \%$ & $81 \%$ & $19 \%$ \\
\hline 2.- ¿Se preocupa de que su hijo-alumno use un calzado adecuado? & $78 \%$ & $22 \%$ & $63 \%$ & $37 \%$ \\
\hline 3.- ¿Tiene conocimiento sobre pie plano? & $65 \%$ & $35 \%$ & $63 \%$ & $37 \%$ \\
\hline 4.- ¿Tiene conocimiento sobre pie cavo? & $46 \%$ & $54 \%$ & $37 \%$ & $63 \%$ \\
\hline $\begin{array}{l}\text { 5.- ¿Conoce Ud. si existe preocupación de parte de los Servicios } \\
55 \% \text { de Salud, sobre anomalías de pie? }\end{array}$ & $26 \%$ & $74 \%$ & $25 \%$ & $75 \%$ \\
\hline $\begin{array}{l}\text { 6.- ¿Conoce Ud. las consecuencias que puede provocar no detectar y } \\
\text { tratar a tiempo estas anomalías? }\end{array}$ & $55 \%$ & $45 \%$ & $50 \%$ & $50 \%$ \\
\hline $\begin{array}{l}\text { 7.- ¿Sabe Ud. si las plantillas son el único tratamiento para tratar pie } \\
\text { plano y pie cavo? }\end{array}$ & --- & --- & $22 \%$ & $78 \%$ \\
\hline $\begin{array}{l}\text { 8.- ¿Ac eptaría el apoyo de un especialista en las clases de } \\
\text { Educación Física? }\end{array}$ & --- & --- & $100 \%$ & --- \\
\hline
\end{tabular}

\section{DISCUSIÓN}

En el transcurso del crecimiento y desarrollo humano se suceden variados y determinantes cambios, quizás uno de los más importantes lo representa el lo- gro de la postura erguida y la expresión de la locomoción, característica fundamental de la especie humana (Didia). 
La estructura del pie y la consolidación del arco plantar se expresa en la primera etapa del desarrollo infantil ( 0 a 5 años) y se estima que este proceso termina entre los 6 a los 12 años.

El transcurso por la etapa escolar permite detectar una serie de alteraciones en el aparato locomotor, según la edad de los niños, sobre todo en el pie, que mayoritariamente se clasifica en base a la altura del arco y el alineamiento del retropié y el antepié en, pie cavo (arco elevado), neutral alineados (normal) y pie plano o de arco bajo (Razeghi \& Batt, 2002; Nikolaidou \& Boudolos; Onodera et al.).

Este estudio analiza la prevalencia de pie plano y pie cavo en población escolar de entre 6 a 12 años, incorporando además las variables del peso corporal y el tipo de establecimiento educacional.

La literatura informa que pie plano, con disminución de la bóveda plantar y desviación del talo en valgo, es una condición fisiológica durante los 3 primeros años de vida, luego el arco plantar se eleva, por lo que no se requeriría tratamiento podológico. En cambio el pie cavo presentaun aumento de la altitud y amplitud del arco plantar, donde el retropié y el antepié están más próximos, habitualmente asintomático, pero puede causar cansancio o dolor en el pie (Larrosa Padró \& Mas Moline).

En la Tabla I se observa que los niños presentan mayor peso que las niñas (Malina et al., 2004). Los índices de masa corporal en esta población en estudio muestra similar comportamiento, según los resultados obtenidos por Carrasco et al. (2011), en estudiantes de colegios municipalizados de la Región de Temuco.

El índice de masa corporal (IMC), en los niños a partir de los 7 años presentan valores que indican sobrepeso muy similar a lo reportado en escolares de Taiwán, donde los hombres con pie plano presentan una alta correlación con sobrepeso y obesidad (WHO, 2009; Villarroya et al., 2009; Chang et al., 2010; Chen et al., 2011).

Las Tablas II y III muestran la prevalencia de las anomalías de pie según sexo y edad, e indican que pie plano tiene mayor expresión en los niños que en las niñas, con un promedios generales de $31,6 \%$ y $24,3 \%$ respectivamente. Estos resultados son coincidentes a los observados por Pauk et al. (2010), en niños de Polonia, donde las niñas presentan una mayor elevación del arco plantar respecto a los niños en estudio. En otro estudio realizado en población turca, se determinan valores de $35,5 \%$ para las niñas y de 28,5\% para los niños (Cetin et al., 2011). Similares resultados describe Aminian \& Sangeorzan (2008), con mayor prevalencia de pie cavo en las niñas con $14,4 \%$ versus $11,6 \%$ de los niños.

La mayoría de los autores coinciden en que determinar prevalencia de anomalías de pie resulta muy difícil, debido sobre todo a los diferentes métodos de diagnóstico con variaciones que expresan valores que oscilan desde un $2,7 \%$ a un $70-80 \%$, dependiendo de las metodologías utilizadas, ya sea por simple observación o por alta tecnología de resonancia magnética. Otros autores determinan que posiblemente los mejores registros serían los entregados por la medición de la altura navicular y la huella plantar, con lo cual se podría implementar un modelo universal de evaluación, según, sexo, edad y grupo etáreo. Lo anterior para aplicar mejores tratamientos fisioterápicos globales (Rosenberg \& Zanetti, 2005; Ballesteros, 2010; Evans et al., 2011).

El medio socioeconómico, lugar de residencia, el uso de calzado, la preocupación del núcleo familiar y estudiantil, también resultan factores importante al momento de establecer prevalencia poblacional, como se muestra en las Tablas IV y V, donde las mayores anomalías de pie se observan en los alumnos de colegio municipal, público y gratuito, con un $45 \%$ de incidencia, cuyos padres presentan un nivel socioeconómico más bajo. Los mejores índices de normalidad de pie, se observan en los colegios particular y particular subvencionado (57\% y $65 \%$ respectivamente), con padres de altos índices socioeconómico, lo cual posibilitaría el uso de calzado más adecuado, pero más oneroso. Pocos estudios se refieren a la relación entre el nivel socio económico el uso de calzado adecuado y las anomalías de pie, Crosbie \& Burns (2008) asocian el uso incorrecto del calzado a una magnificación del dolor en pie cavo, debido a que provocaría un patrón de marcha mas cauteloso, con mayor presión en el retropié y antepié. Cetin et al. encontraron que los niños de zonas rurales que solo usan sandalias tienen menores incidencias de pie plano que los niños de centros urbanos que usan calzado cerrado.

El presente estudio se direcciona a determinar las prevalencias de las anomalías de pie paraimplementar mejores políticas de prevención y corrección, ya que muchas alteraciones podológicas pueden evolucionar a trastornos más severos, alterando la calidad de vida de los niños en la marcha, el equilibrio y la actividad física.

\section{CONCLUSIONES}

El pie plano presenta alta prevalencia en la población estudiantil de Arica, del $28 \%$, y pie cavo una prevalencia del $13 \%$. Los niños, presentan una prevalencia de un $31,6 \%$ para 
pie plano y un $11.6 \%$ para pie cavo, y laas niñas presentan una prevalencia de $24,3 \%$ y $14,4 \%$, respectivamente.

El peso y el IMC presentan una correlación positiva en la expresión de anomalías de pie en la población estudiantil de Arica-Chile.

Según el tipo de establecimiento escolar, el Colegio "Regimiento Rancagua", de tipo municipal, gratuito, presenta los más altos índices de anomalías de pie, con una incidencia de un $45 \%$. Los Colegios particular subvencionado y Particular, presentan los mejores índices de normalidad (35\% y $43 \%$ respectivamente).

Los padres a apoderados tienen pocos conocimientos sobre estas alteraciones y sus consecuencias en la postura. Pie plano es la anomalía de mayor conocimiento por padres y los profesores.

Se sugiere mayor estudio de estas temáticas, para reconocer y detectar estas anomalías posturales, que necesita tratamiento del especialista en forma precoz.

ESPINOZA-NAVARRO, O.; OLIVARES, U. M.; PALACIOS, N. P. \& ROBLES, F. N. Prevalence of foot anomalies in schoolchildren between 6 and 12 years old of elementary education from Arica-Chile. Int. J. Morphol., 31(1):162-168, 2013.

SUMMARY: Orthopedic foot problems in children are the most common at this time of life. For this reason it is important to differentiate between a normal foot and an altered foot. The aim of this study was to determine the prevalence of flat and cavus foot in elementary schoolchildren to Arica, Chile. Four hundred and twenty students (210 girls and 210 boys) from 3 different schools of elementary education in the city of Arica, ages 6-12 years, apparently healthy, were measured. All study subjects underwent an assessment of the footprint from the anteroposterior plane, using a podograph. Parents and teachers were surveyed about the level of knowledge and the implications of these anomalies. The results show in boys a prevalence of $31.6 \%$ for flat foot and $11.6 \%$ for cavus foot. In girls the prevalence was $24.3 \%$ to flat foot and $14.4 \%$ for cavus foot. The weight and body mass index are positively correlated in expression standing anomaly in the student population of Arica, Chile. The public, municipal and gratuitous schools, have the highest standing indices of anomalies, with values of $45 \%$. The private schools $(43 \%)$, subsidized private schools, showing lower rates of abnormal foot, with $35 \%$. In the analysis of the survey shows that few parents have knowledge of these changes and their consequences for the position. Flat foot is the anomaly of greater awareness by parents and teachers. We conclude that flatfoot is more prevalent in the student population of Arica, with $28 \%$, cavus foot presenting a prevalence of $13 \%$.

KEY WORDS: Postural alterations; Elementary education, Arica-Chile; Children.

\section{REFERENCIAS BIBLIOGRÁFICAS}

Aminian, A. \& Sangeorzan, B. The anatomy of cavus foot deformity. Foot Ankle Clin., 13(2):191-8, 2008.

Ballesteros, R. Efectividad del abordaje fisioterápico global a través del método G.D.S. en el pie plano flexible infantil. REDUCA (Enfermería, Fisioterapia y Podología), 2(1):96$114,2010$.

Carrasco, V.; Mártínez, C.; Silva, H.; Collipal, E.; Campos, C. \& Silva, T. Prevalence of obesity in a sample of schoolchildren from municipalzed school in the IX Región of Chile 2008-2009. Int. J. Morphol., 29(3):830-4, 2011.

Cetin, A.; Sevil, S.; Karaoglu, L. \& Yucekaya, B. Prevalence of flat foot among elementary school students, in rural and urban areas and at suburbs in Anatolia. Eur. J. Orthop. Surg. Traumatol., 21(5):327-31, 2011.

Comín, M.; Pérez, J. \& Villarroya, A. Factores que influyen en las presiones plantares. Med. Rehab., XII(3):31-39, 1999.

Chang, J. H.; Wang, S. H.; Kuo, C. L.; Shen, H. C.; Hong, Y. W. $\&$ Lin, L. C. Prevalence of flexible flatfoot in Taiwanese school-aged children in relation to obesity, gender and age. Eur. J. Pediatr., 169(4):447-52, 2010.

Crosbie, J. \& Burns, J. Are in-shoe pressure characteristics in symptomatic idiopathic pes cavus related to location of foot pain? Gait Posture, 27(1):16-22, 2008.

Chen, K. C.; Yeh, C. J.; Tung, L. C.; Yang, J. F.; Yang, S. F. \& Wang, C. H. Relevant factors influencing flatfoot in preschool-aged children. Eur. J. Pediatr., 170(7):931-6, 2011.

Didia, B. C. Man: Know Thyself. Inaugural lecture series. College of Health Science. Port Harcourt, Nigeria, University of Port Harcourt, 2011.

El, O.; Akcali, O.; Kosay, C.; Kaner, B.; Arsian, Y.; Sagoi, E.; et al. Flexible flatfoot and related factors in primary school children: a report of a screening study. Rheumatol. Int., 26(11):1050-3, 2006.

Evans, A.; Scutter, S. \& Kneebone, K. Clinical measures of paediatric foot posture: a critical review. J. Foot Ankle Res., 4(Suppl 1):P20, 2011.

Ibañez, A.; Baar, A. \& Gana, N. Cambios fisiológicos de la rotación de la marcha durante el desarrollo. Rev. Chil. Pediatr., 79(1):45-9, 2008.

Ledoux, W. R.; Rohr, E. S.; Ching, R. P. \& Sangeorzan, B. J. Effect of foot shape on the three-dimensional position of foot of bones. J. Orthop. Res., 24(12):2176-86, 2006. 
ESPINOZA-NAVARRO, O.; OLIVARES, U. M.; PALACIOS, N. P. \& ROBLES, F. N. Prevalencia de anomalías de pie en niños de enseñanza básica de entre 6 a 12 años, de colegios de la ciudad de Arica-Chile. Int. J. Morphol., 31(1):162-168, 2013.

Larrosa Padró, M. \& Mas Moliné, S. Alteraciones de la bóveda plantar. Rev. Esp. Reumatol., 30(9):489-98, 2003.

Malina, R. M.; Bouchard, C. \& Bar-Or, O. Growth, maturation, and physical activity. $2^{\text {nd }}$ ed. Champaign, IL, Human Kinetics, 2004. pp.101-17.

Ministerio de Salud de Chile (Minsal). Norma técnica de evaluación nutricional del niño de 6 a 18 años. Rev. Chil. Nutr., 31(2):128-37, 2004

Nikolaidou, M. \& Boudolos, K. A footprint-based approach for the rational classification of foot types in young schoolchildren. Foot, 16(2):82-90, 2006.

Onodera, A.; Neves, I.; Morioka, E.; Souza, P.; Sá, M. \&; Amadio, A. What is the best method for child longitudinal plantar arch assessment and when does arch maturation occur? Foot, 18(3):142-9, 2008.

Pauk, J.; Ezerskiy, V. \& Rogalski, M. Impact of epidemiological factors on occurrence of platypodia in children. Fizjoterapia, 18(2):21-7, 2010.

Razeghi, M. \& Batt, M. Foot type classification: a critical review of current methods. Gait Posture, 15(3):282-91, 2002.

Rosenberg, Z. S. \& Zanetti, M. Imaging of the foot and ankle. In: Hodler, J.; Zollikofer, Ch. L. \& von Schulthess, G. K. (edr.). Musculoskeletal Diseases. IDKD 2005. Milan, Springer Milan, 2005. pp.39-47.

Vasconcellos, H.; Cavalcante, M.; Ferreira, I.; Frango, A.; Siqueira, C. \& Galvao, J. El cambio de forma del músculo aductor hallux y el halux valgus. Int. J. Morphol., 29(4):1303-6, 2011.

Viladot, R. \& Rochera, R. Pie zambo. En: Viladot Voegli, A. \& Viladot Pericé, R. (edr.). 20 lecciones sobre patología del pie. Barcelona, Ediciones Mayo, 2009. pp.39-59.

Villarroya, M. A.; Esquivel, J. M.; Tomás, C.; Moreno, L. A.; Buenafé, A. \& Bueno, G. Assessment of the medial longitudinal arch in children and adolescents with obesity: Footprints and radiographic study. Eur. J. Pediatr., 168(5):559-67, 2009.

World Health Organization (WHO). Health topics: Obesity, 2009. Disponible en: http://www.who.int/topics/obesity/

Zurita, M. F. \& Cabello, M. D. Influencia del pie en la estática, marcha y otras habilidades en escolares de 6 a 12 años. Lect. Educ. Fís. Deportes (B. Aires), 8(51), 2002. Disponible en: http://www.efdeportes.com/efd51/pie.htm
Dirección para correspondencia:

Prof. Dr. Omar Espinoza Navarro

Facultad de Ciencias, Departamento de Biología

Universidad de Tarapacá

Avenida. General Velásquez Nº 1775

Arica

CHILE

Fono: 5658205415

Fax: 5658205381

Email: oespinoz@uta.cl

Recibido : 24-09-2012

Aceptado: 21-10-2012 